\title{
E-PASS Scoring System May Be Useful for Prediction of Postoperative Complications in Super Elderly Colorectal Cancer Surgery Patients
}

\author{
Hiroka Kondo, Yasumitsu Hirano, Toshimasa Ishii, Kiyoka Hara, Nao Obara, Liming Wang, Masahiro Asari, \\ Takuya Kato and Shigeki Yamaguchi
}

Department of Gastroenterological Surgery, Saitama Medical University International Medical Center, Hidaka, Japan

\begin{abstract}
Objectives: Japan is facing an aging society. Elderly individuals are generally more prone to comorbidities and have weaker immune defenses, with ominous prognostic implications if postoperative complications arise. The aim of this study was to explore scoring systems for predicting postoperative morbidity risk in super elderly patients ( $\geq 85$ years old) after colorectal surgery for cancer.

Methods: A population of elderly patients $(n=145)$ surgically treated for primary colorectal cancer within our department between April 2007 and December 2018 was examined retrospectively, assessing the capacities of various indices, such as Estimation of Physiologic Ability and Surgical Stress (E-PASS), neutrophil/ lymphocyte ratio (NLR), platelet/lymphocyte ratio (PLR), prognostic nutritional index (PNI), and modified Glasgow Prognostic Score (mGPS), to predict postoperative complications.

Results: NLR, PLR, and mGPS did not differ significantly in the presence or absence of complications, whereas PNI tended to be lower if complications developed. The E-PASS system showed no group-wise differences in preoperative risk score (PRS), but the surgical stress score (SSS) and the comprehensive risk score (CRS; a composite of PRS and SSS) was significantly higher in patients with complications. Based on the cutoff value calculated from the Receiver operating curve (ROC) for the E-PASS CRS $(-0.0580$ ), patients were stratified into low-scoring and high-scoring (HSG) groups. Although not significantly different, the overall survival in the HSG tended to be lower by comparison.

Conclusions: The E-PASS scoring system may be a useful predictor of postoperative complications in super elderly patients requiring colorectal cancer surgery.
\end{abstract}

\section{Keywords}

colorectal cancer, elderly, postoperative complications, prognostic scoring

J Anus Rectum Colon 2020; 4(3): 137-144

\section{Introduction}

In Japan, the average life expectancy has increased each year, having reached 80.77 years for men and 87.01 years for women by 2015[1]. Patients beyond 85 years of age are not uncommon at our hospital. Elderly people are typically plagued by more comorbidities and weaker immune systems, so postoperative complications may have devastating prognostic implications. Appropriate risk assessment is essential in their surgical treatment. The aim of this study was to explore scoring systems for predicting postoperative morbidity risk in super elderly patients ( $\geq 85$ years old) surgically treated for colorectal cancer. 


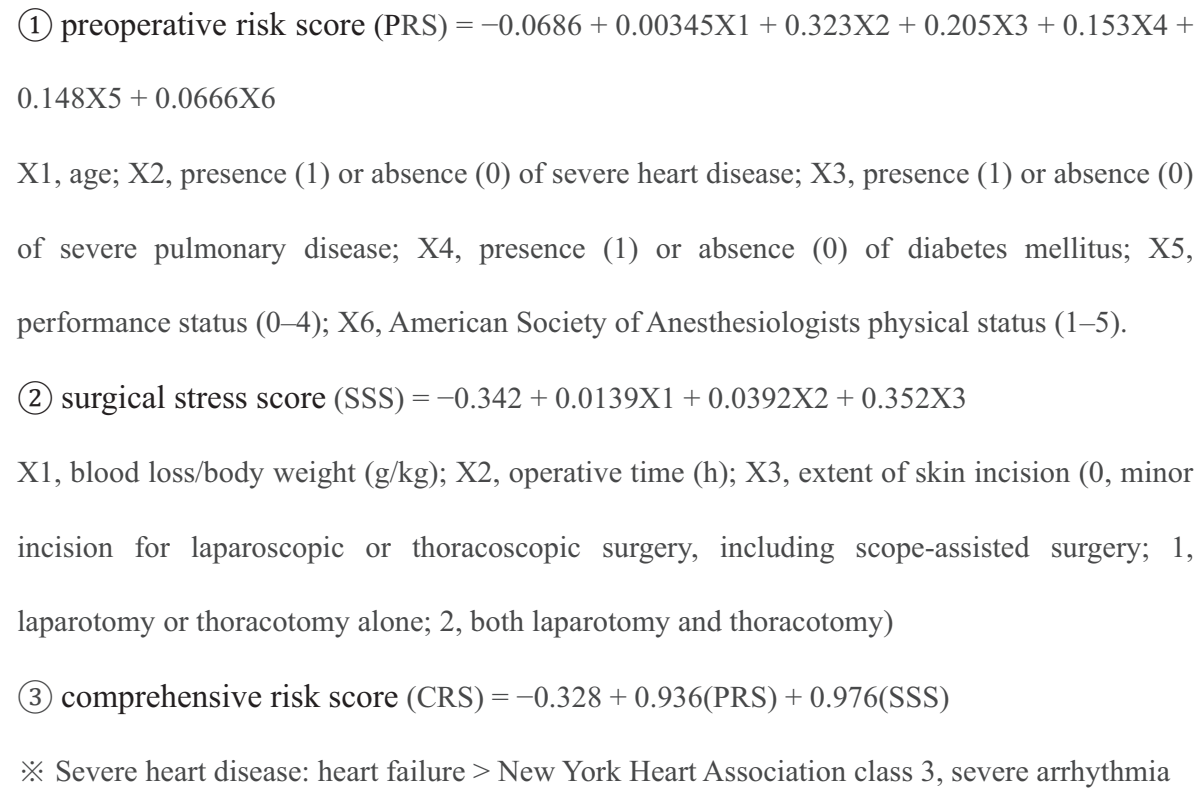

※ Severe pulmonary disease: \%Vital Capacity $<60 \%$ or FEV $1 \%<50 \%$

Figure 1. Estimation of Physiologic Ability and Surgical Stress.

Table 1. Glasgow Prognostic Score (GPS) and Modified GPS (mGPS).

\begin{tabular}{cc}
\hline GPS & Score \\
\hline $\mathrm{CRP} \leq 1.0 \mathrm{mg} / \mathrm{dL}$ and $\mathrm{ALB} \geq 3.5 \mathrm{~g} / \mathrm{dL}$ & 0 \\
$\mathrm{CRP}>1.0 \mathrm{mg} / \mathrm{dL}$ or $\mathrm{ALB}<3.5 \mathrm{~g} / \mathrm{dL}$ & 1 \\
$\mathrm{CRP}>1.0 \mathrm{mg} / \mathrm{dL}$ and $\mathrm{ALB}<3.5 \mathrm{~g} / \mathrm{dL}$ & 2 \\
\hline $\mathrm{mGPS}$ & Score \\
\hline $\mathrm{CRP} \leq 0.5 \mathrm{mg} / \mathrm{dL}$ and $\mathrm{ALB} \geq 3.5 \mathrm{~g} / \mathrm{dL}$ & 0 \\
$\mathrm{CRP}>0.5 \mathrm{mg} / \mathrm{dL}$ or ALB $<3.5 \mathrm{~g} / \mathrm{dL}$ & 1 \\
$\mathrm{CRP}>0.5 \mathrm{mg} / \mathrm{dL}$ and $\mathrm{ALB}<3.5 \mathrm{~g} / \mathrm{dL}$ & 2 \\
\hline
\end{tabular}

CRP, C-reactive protein; ALB, albumin

\section{Methods}

A population of elderly patients $(\mathrm{n}=145)$ submitting to primary colorectal cancer resection within our department between April 2007 and December 2018 were subjected to retrospective review. Emergency surgical procedures were grounds for exclusion, as were dual or multiple cancers. In the study participants, we examined the capacities of certain indices, such as Estimation of Physiologic Ability and Surgical Stress (E-PASS), neutrophil/lymphocyte ratio (NLR), platelet/lymphocyte ratio (PLR), prognostic nutritional index (PNI), and modified Glasgow Prognostic Score (mGPS), to predict postoperative complications.

E-PASS was first proposed by Haga et al. as a standard method of surgical risk assessment (Figure 1)[2]. The preoperative risk score (PRS) of E-PASS addresses the physi- ological state, whereas the surgical stress score (SSS) gauges the impact of surgical penetration. The comprehensive risk score (CRS) involves both PRS and SSS; the higher the score is, the greater the risk. NLR and PLR have also been identified as prognostic factors in various types of cancer, higher scores again denoting poorer prognoses[3,4]. The PNI of Onodera et al. was originally intended for predicting risks of perioperative complications. PNI is calculated by the following formula: $10 \times$ serum albumin $(\mathrm{g} / \mathrm{dL})+0.005 \times$ total lymphocytes/ $\mu \mathrm{L}$. A PNI score of $\leq 40$ is an acknowledged contraindication for anastomosis[5]. C-reactive protein (CRP) and serum albumin are incorporated in the GPS. Jiang et al. (2015) have previously determined its prognostic value in patients with non-small cell lung cancer[6]. The mGPS (Table 1) was formulated in Japan by Miki et al., setting a CRP cutoff value of 0.5 . This index has proven useful in predicting colorectal cancer outcomes[7], a higher score signaling a poor prognosis. This was a retrospective study. For statistical analysis, we performed chi-squared and Mann-Whitney $U$ tests to examine the differences between the two groups. In order to control for confounding factors, binary logistic regression was used. The cumulative survival rate was analyzed with Kaplan-Meier and log-rank tests. P < 0.05 was considered a statistically significant difference.

The Ethics Committee of the Saitama Medical University International Medical Center approved the study that was conducted in accordance with the Helsinki Declaration of 1996. Also, a written consent for the publication of this article was obtained from the patients. The consent was written in Japanese for better understanding by the patient. The con- 
sent form will be provided to the editors of this journal on request.

Table 2. Characteristics of the Patient Population.

\begin{tabular}{ll}
\hline Male:female & $72: 73$ \\
Age, mean (range) & $87.8 \mathrm{yrs}(85-97 \mathrm{yrs})$ \\
ASA 2/ASA 3 & $126 / 19$ \\
Laparoscopy/laparotomy & $109 / 36$ \\
Stage 0/1/2/3/4 & $1 / 32 / 57 / 44 / 11$ \\
Operative time, mean (range) & $175.3 \mathrm{~min}(32-482 \mathrm{~min})$ \\
Blood loss, mean (range) & $60 \mathrm{~mL}(0-974 \mathrm{~mL})$ \\
Postoperative complication & \\
Clavien-Dindo Grade I-V & 34 cases $(23.4 \%)$ \\
\hline
\end{tabular}

ASA, American Society of Anesthesiologists

\section{Results}

Within our department, 145 patients (men, 72; women, 73 ), each $\geq 85$ years old and requiring resection of colorectal cancer, were selected for the study. As shown in Table 2, laparoscopic surgery accounted for $\sim 75 \%$ of the procedures. Complications of any magnitude (Clavien-Dindo Grades IV) developed in $23.4 \%(34 / 145)$ of patients. A detailed analysis of patients with (+) or without (-) complications is provided in Table 3. They did not differ significantly in age, sex, American Society of Anesthesiologists (ASA) physical status, preoperative carcinoembryonic antigen (CEA) level, or presence/absence of severe heart disease, lung disease, or diabetes. However, the body mass index (BMI) showed a slight downward tendency in the presence of complications; and in the group without complications, significantly more laparoscopic surgeries took place. Tumor locations, operative times, and blood loss volumes were similar for the two

Table 3. Univariate Analysis of Patients in Terms of Postoperative Complications.

\begin{tabular}{|c|c|c|c|}
\hline Variable & $\begin{array}{l}\text { Complication }(+) \\
(\mathrm{n}=34)\end{array}$ & $\begin{array}{l}\text { Complication }(-) \\
\quad(\mathrm{n}=111)\end{array}$ & $P$-value \\
\hline Age (years) & $87(85-94)$ & $87(85-97)$ & NS \\
\hline Sex (male/female) & $16 / 18$ & $56 / 55$ & NS \\
\hline ASA 2/ASA 3 & $29 / 5$ & $97 / 14$ & NS \\
\hline CEA & $4.3(1.2-111.3)$ & $4.8(0.9-2426.6)$ & NS \\
\hline BMI & $20.6(16.3-29.0)$ & $21.6(14.9-33.3)$ & 0.081 \\
\hline Heart disease (yes/no) & $1 / 33$ & $1 / 110$ & NS \\
\hline Pulmonary disease (yes/no) & $2 / 32$ & $2 / 109$ & NS \\
\hline Diabetes mellitus (yes/no) & $5 / 29$ & $18 / 93$ & NS \\
\hline Laparoscopic surgery (yes/no) & $21 / 13$ & $88 / 23$ & 0.039 \\
\hline Tumor location (C/A/T/D/S/R) & $1 / 8 / 3 / 3 / 7 / 12$ & $16 / 30 / 12 / 6 / 26 / 21$ & NS \\
\hline TNM stage (0/I/II/III/IV) & $0 / 5 / 11 / 14 / 4$ & $1 / 27 / 46 / 30 / 7$ & NS \\
\hline Operative time (min) & $182(32-329)$ & $160(52-482)$ & NS \\
\hline Blood loss (mL) & $15(0-642)$ & $5(0-974)$ & NS \\
\hline \multicolumn{4}{|l|}{ Surgical risk scoring system } \\
\hline E-PASS PRS & $\begin{array}{c}0.5098 \\
(0.3579-1.2034)\end{array}$ & $\begin{array}{c}0.5059 \\
(0.3579-1.1098)\end{array}$ & NS \\
\hline E-PASS SSS & $\begin{array}{c}-0.1515 \\
(-0.2600-0.4233)\end{array}$ & $\begin{array}{c}-0.2035 \\
(-0.2990-0.5933)\end{array}$ & 0.005 \\
\hline E-PASS CRS & $\begin{array}{c}0.0919 \\
(-0.2400-0.5623)\end{array}$ & $\begin{array}{c}-0.0484 \\
(-0.257-0.8788)\end{array}$ & 0.018 \\
\hline NLR & $\begin{array}{c}2.687 \\
(0.9483-12.608)\end{array}$ & $\begin{array}{c}2.777 \\
(1.0453-16.336)\end{array}$ & NS \\
\hline PLR & $\begin{array}{c}216.2 \\
(60.818-487.18)\end{array}$ & $\begin{array}{c}174.4 \\
(53.939-718.21)\end{array}$ & NS \\
\hline PNI & $\begin{array}{c}42.415 \\
(22.375-53.525)\end{array}$ & $\begin{array}{c}44.295 \\
(25.005-57.81)\end{array}$ & 0.076 \\
\hline mGPS $(0$ and $1 / 2)$ & $32 / 2$ & $108 / 3$ & NS \\
\hline
\end{tabular}

ASA, American Society of Anesthesiologists; CEA, carcinoembryonic antigen; BMI, body mass index; C/ A/T/D/S/R: cecum/ascending/transverse/descending/sigmoid/rectum; E-PASS, Estimation of Physiologic Ability and Surgical Stress; PRS, preoperative risk score; SSS, surgical stress score; CRS, comprehensive risk score; NLR, neutrophil/lymphocyte ratio; PLR, platelet/lymphocyte ratio; PNI, prognostic nutritional index; mGPS, modified Glasgow Prognostic Score 
groups. In the scoring of surgical risk, no significant groupwise differences in NLR, PLR, or mGPS emerged, although a tendency for slightly lower PNI was shown by patients with complications. In terms of E-PASS, the PRS values did not differ significantly in the two groups, but the SSS and CRS (a composite of PRS and SSS) values were significantly higher in the group with complications.

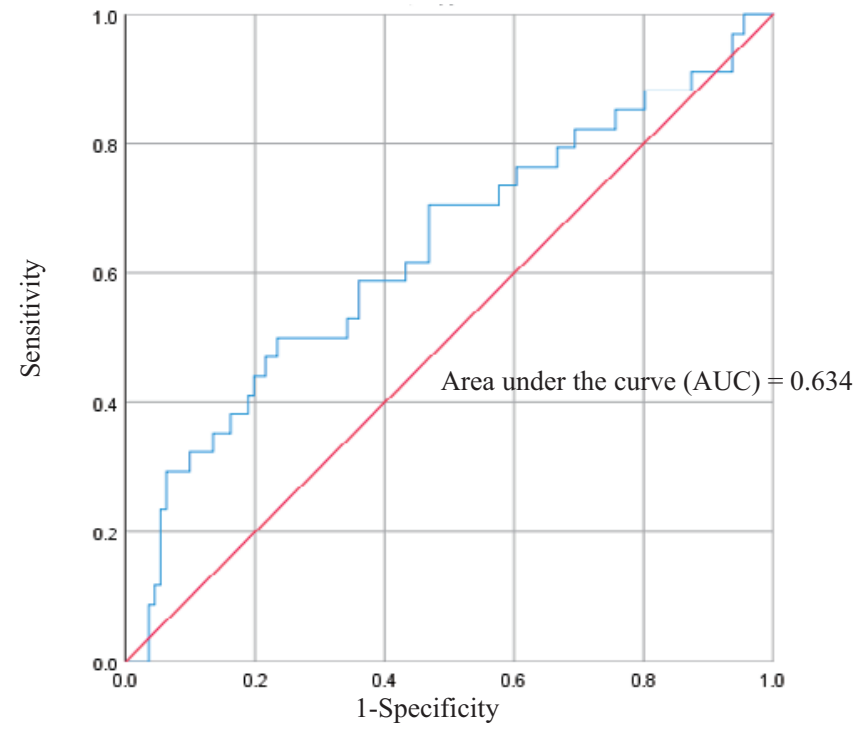

Figure 2. Receiver operating curve analysis for postoperative complications. Area under the curve (AUC) of E-PASS (CRS) is 0.634 .
We determined by ROC analysis CRS $=-0.0580$ as the cutoff value for the E-PASS CRS. The sensitivity and specificity of CRS $=-0.0580$ was 0.706 and 0.532 , respectively. The area under the curve (AUC) of the model for E-PASS CRS was 0.634 (Figure 2). Based on this cutoff value for the E-PASS CRS (-0.0580), we also assigned patients to low-scoring (LSG, $\mathrm{n}=69$ ) and high-scoring (HSG, $\mathrm{n}=76$ ) groups for analysis of short-term postoperative results (Table 4). These groups did not differ significantly in age, sex, preoperative CEA level, BMI, or presence/absence of severe heart or lung disease. As a matter of course (inherent to the CRS formula), the ASA 2/ASA 3 ratio, performance status, and diabetes comorbidity rate were high in the HSG. However, laparoscopic surgery was performed more frequently in LSG members. No significant group differences in tumor locations and TNM stages were evident. Although the operative times of the two groups were similar, blood loss was greater in the HSG. HSG members had a higher postoperative complication rate than LSG members, and the length of postoperative hospital stay was longer. The survival curves of both groups are plotted in Figure 3. Survival was somewhat lower for HSG (vs. LSG) members, falling short of significance. In multivariate analysis, the HSG was associated with a higher chance of developing complications (odds ratio $[\mathrm{OR}] 2.776,95 \%$ confidence interval $[95 \% \mathrm{CI}] 1.214-$ 6.351, $\mathrm{p}=0.016$ ) (Table 5).

It is self-evident that laparoscopic surgery results in lower SSS and CRS. Therefore, we further examined whether E-

Table 4. Univariate Analysis of Patients Stratified by E-PASS Comprehensive Risk Score (CRS).

\begin{tabular}{lccc}
\hline \multicolumn{1}{c}{ Variable } & $\begin{array}{c}\text { Low-scoring group } \\
(\mathrm{n}=69)\end{array}$ & $\begin{array}{c}\text { High-scoring group } \\
(\mathrm{n}=76)\end{array}$ & $P$-value \\
\hline Age (years) & $87(85-94)$ & $87(85-97)$ & $\mathrm{NS}$ \\
Sex (male/female) & $33 / 36$ & $39 / 37$ & $\mathrm{NS}$ \\
ASA 2/ASA 3 & $66 / 3$ & $60 / 16$ & 0.003 \\
Peformance status (0/1-4) & $54 / 15$ & $28 / 48$ & $<0.001$ \\
CEA & $4.5(1.2-2426.6)$ & $4.8(0.9-111.3)$ & $\mathrm{NS}$ \\
BMI & $21.83(15.7-33.3)$ & $21.4(14.9-29.3)$ & $\mathrm{NS}$ \\
Heart disease (yes/no) & $0 / 69$ & $2 / 74$ & $\mathrm{NS}$ \\
Pulmonary disease (yes/no) & $0 / 69$ & $4 / 72$ & 0.073 \\
Diabetes mellitus (yes/no) & $6 / 63$ & $17 / 59$ & 0.024 \\
Laparoscopic surgery (yes/no) & $69 / 0$ & $40 / 36$ & 0.0001 \\
Tumor location (C/A/T/D/S/R) & $9 / 17 / 7 / 3 / 18 / 15$ & $8 / 20 / 9 / 6 / 15 / 18$ & $\mathrm{NS}$ \\
TNM stage (0//II/III/IV) & $0 / 18 / 27 / 21 / 3$ & $1 / 14 / 30 / 23 / 8$ & $\mathrm{NS}$ \\
Operative time (min) & $153(82-290)$ & $180(32-482)$ & $\mathrm{NS}$ \\
Blood loss (mL) & $5(0-302)$ & $30(0-974)$ & 0.0001 \\
Postoperative complication (+/-) & $10 / 59$ & $24 / 52$ & 0.015 \\
& $14.49 \%$ & $31.58 \%$ & \\
Postoperative hospital stay & $7(5-16)$ & $8(4-86)$ & 0.005 \\
\hline
\end{tabular}

ASA, American Society of Anesthesiologists; CEA, carcinoembryonic antigen; BMI, body mass index; C/A/ T/D/S/R: cecum/ascending/transverse/descending/sigmoid/rectum; E-PASS, Estimation of Physiologic Ability and Surgical Stress 


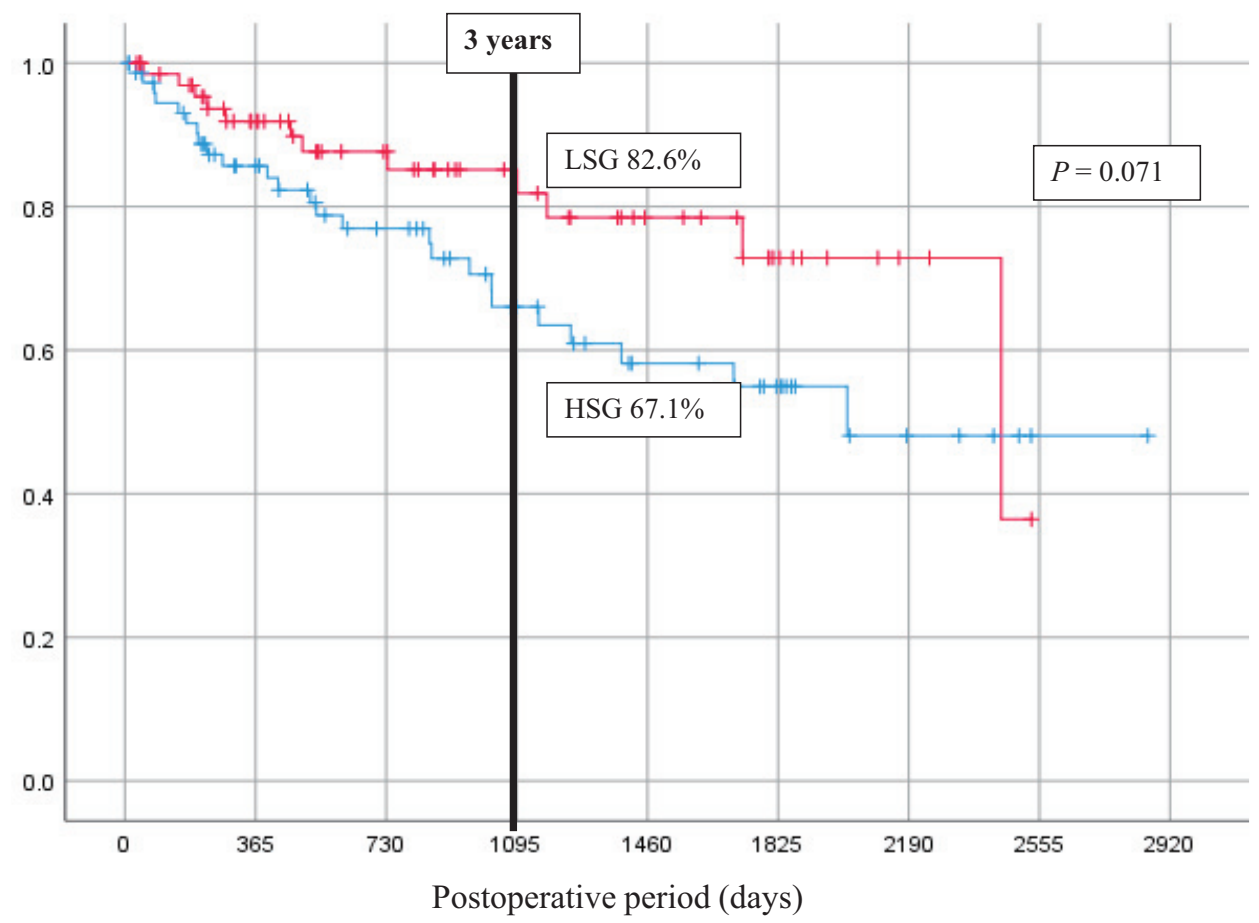

E-PASS, Estimation of Physiologic Ability and Surgical Stress; CRS, comprehensive risk score; HSG, high-scoring group; LSG, low-scoring group

Figure 3. Overall survival plotted by E-PASS CRS: tendency for lower survival in HSG (vs. LSG).

Table 5. Multivariate Logistic Regression Analysis Evaluating Possible Risk Factors Associated with Complication.

\begin{tabular}{lcccc}
\hline & $\begin{array}{c}\text { Partial regression } \\
\text { coefficient }\end{array}$ & Odds ratio & $\begin{array}{c}95 \% \text { confidence } \\
\text { interval }\end{array}$ & $P$-value \\
\hline CRS (HSG vs LSG) & 1.021 & 2.776 & $1.214-6.351$ & 0.016 \\
constant & -1.775 & 0.169 & & 0.000 \\
\hline
\end{tabular}

Model chi-squared test, $\mathrm{p}<0.05$

CRS, comprehensive risk score; HSG, high-score group; LSG, low-score group

PASS is effective even in open surgery. We determined by ROC analysis CRS $=0.2955$ as the cutoff value for the EPASS CRS. The sensitivity and specificity of CRS $=0.2955$ was 0.615 and 0.261 , respectively. The AUC of the model for E-PASS CRS was 0.605 (Figure 4). Based on this, we also assigned patients to LSG $(n=22)$ and HSG $(n=14)$ for analysis of short-term postoperative results (Table 6). As with the previous study (the first examination including laparoscopy and open surgery), the HSG had poor performance status. Although there was no difference in the operation time between the two groups, the amount of bleeding was higher in the HSG. The postoperative complication rate was higher in the HSG than in the LSG $(57.14 \%$ vs. $22.73 \%$ ). As a result, the length of postoperative hospital stay was longer in the HSG.

\section{Discussion}

In the realm of colorectal or gastric cancer surgery, much of the evidence indicates that elderly individuals are disadvantaged, showing higher postoperative complication and perioperative death rates than a younger population due to comorbidities[8-10]. Still, the premise that advanced age itself is a risk factor has been strongly disputed[11-15]. According to Banysch et al., colorectal surgery may be safely performed in patients past the age of 80 if the ASA status is good and there are no grave comorbid conditions[16]. Other sources have also claimed that lower BMI imparts high risk in the elderly population[17], and that low PNI heightens risks of postoperative complications and mortality after colorectal cancer surgery[18,19]. Unfortunately, many ambigui- 
ties exist with regard to the types of patients warranting special perioperative attention and specific measures to reduce surgical risks.

Given these issues, various scoring systems have been used to predict the risk of perioperative complications. Recently, reports have surfaced on the utility of E-PASS in this setting[20-22]. In our study, we examined the super elderly, 85 years of age or older, and tried to identify risk factors

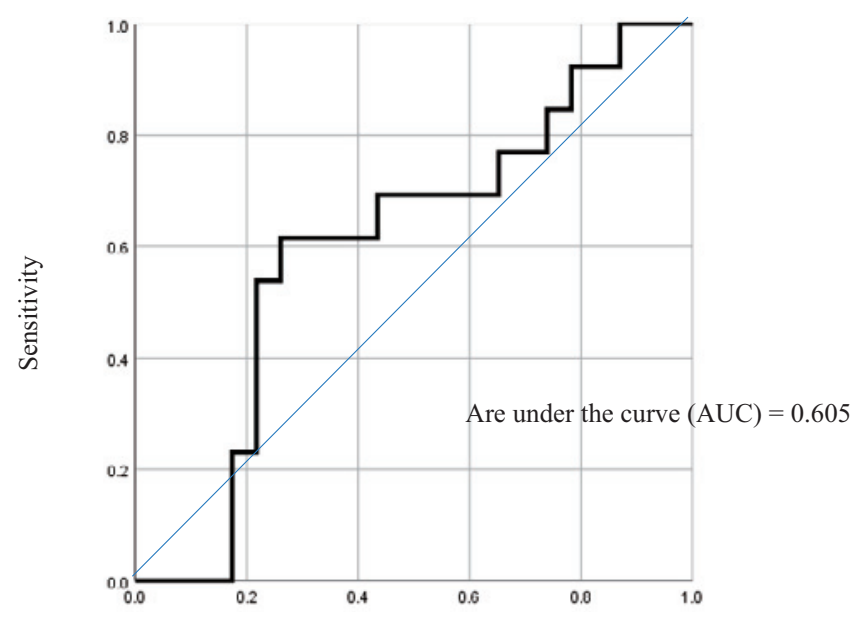

1-Specificity

Figure 4. Receiver operating curve analysis for postoperative complications: focused on only laparotomy. Area under the curve (AUC) of E-PASS (CRS) is 0.605 . other than age, and both SSS and CRS were significantly higher in patients who developed postoperative complications. The frequency of such complications, and PRS values as well, are known to increase with age; but the PRS is a patient-defined factor, difficult for surgeons to control. In order to lower the SSS, a focus on laparoscopic surgery[23] and shorter operative time is needed. Tominaga et al. have identified a CRS cutoff value of 0.2 at which and beyond postoperative complications are more likely[21]. Similarly, Yamamoto et al. have observed worse patient survival at CRS values of $\geq 0.05$ than at values of $<0.05$ [22]. We examined high- and low-scoring patient subsets, based on the cutoff value calculated from the ROC curve for the E-PASS CRS (-0.0580), determining a lower survival rate in highscoring patients. The overall survival rate in LSG was better (but not significantly) than that in HSG ( $p=0.071)$. Furthermore, Dekker et al. seemed to achieve a 1-year survival rate in elderly patients after radical colorectal cancer resection that was on par with survival in young patients, so the care delivered perioperatively and up to 1 year after surgery is critical[24]. This highlights the importance of efforts to lower the SSS and the need for more diligent postoperative management if SSS and CRS metrics are high. To reduce SSS, laparoscopic approach can be followed instead of laparotomy and the amount of blood loss can be controlled as much as possible. However, the universal threshold for CRS could not be determined from this study alone.

Limitation: Owing to the nature of our hospital, extreme comorbidities are seldom encountered, and $\sim 90 \%$ of all

Table 6. Univariate Analysis of Patients Stratified by E-PASS CRS Focused on Only Laparotomy.

\begin{tabular}{lccc}
\hline \multicolumn{1}{c}{ Variable } & $\begin{array}{c}\text { Low-scoring group } \\
(\mathrm{n}=22)\end{array}$ & $\begin{array}{c}\text { High-scoring group } \\
(\mathrm{n}=14)\end{array}$ & $P$-value \\
\hline Age (years) & $86.8(85-97)$ & $86.6(85-93)$ & $\mathrm{NS}$ \\
Sex (male/female) & $11 / 11$ & $9 / 5$ & $\mathrm{NS}$ \\
ASA 2/ASA 3 & $21 / 1$ & $11 / 3$ & $\mathrm{NS}$ \\
Peformance status (0/1-4) & $16 / 6$ & $2 / 12$ & $<0.001$ \\
CEA & $3.7(0.9-85.8)$ & $5.0(2.8-108.4)$ & $\mathrm{NS}$ \\
BMI & $22.0(14.9-29.3)$ & $19.9(18.3-25.4)$ & $\mathrm{NS}$ \\
Heart disease (yes/no) & $0 / 22$ & $0 / 14$ & $\mathrm{NS}$ \\
Pulmonary disease (yes/no) & $0 / 22$ & $1 / 13$ & $\mathrm{NS}$ \\
Diabetes mellitus (yes/no) & $1 / 21$ & $3 / 11$ & $\mathrm{NS}$ \\
Tumor location (C/A/T/D/S/R) & $4 / 4 / 3 / 4 / 5 / 2$ & $2 / 4 / 1 / 0 / 3 / 4$ & $\mathrm{NS}$ \\
TNM stage (0/I/II/III/IV) & $0 / 2 / 13 / 5 / 2$ & $1 / 2 / 6 / 3 / 2$ & $\mathrm{NS}$ \\
Operative time (min) & $121.5(60-256)$ & $144.5(32-462)$ & $\mathrm{NS}$ \\
Blood loss (mL) & $54.5(0-441)$ & $188(5-974)$ & 0.017 \\
Postoperative complication (+/-) & $5 / 17$ & $8 / 6$ & 0.036 \\
& $22.73 \%$ & $57.14 \%$ & \\
Postoperative hospital stay & $7(5-19)$ & $11.5(4-86)$ & 0.025 \\
\hline
\end{tabular}

ASA, American Society of Anesthesiologists; CEA, carcinoembryonic antigen; BMI, body mass index; C/A/T/D/S/R: cecum/ascending/transverse/descending/sigmoid/rectum; E-PASS, Estimation of Physiologic Ability and Surgical Stress; CRS, comprehensive risk score 
surgical procedures are laparoscopic, explaining the low CRS values at baseline. Therefore, the surgery results may be positive, even within the same study of older individuals $>85$ years. It is important to consider that the same elderly individuals aged $>85$ years are not indicated for cancer resection if their general condition is poor; they were not included in this study. A greater number and a higher diversity of patients must be studied going forward to better delineate CRS thresholds.

\section{Conclusion}

Our data suggest that the E-PASS scoring system has potential as a reliable predictor of postoperative complications in super elderly patients subjected to colorectal cancer surgery.

\section{Conflicts of Interest}

There are no conflicts of interest.

\section{Author Contributions}

HK and YH drafted this manuscript under supervision of SY. The other contributors were tasked with content review. All authors have read and approved the final document.

\section{Approval by Institutional Review Board (IRB)}

Approval code: $19-021$

The name of the institutions: Saitama medical university international medical center

\section{Disclaimer}

Shigeki Yamaguchi is one of the Associate Editors of Journal of the Anus, Rectum and Colon and on the journal's Editorial Board. He was not involved in the editorial evaluation or decision to accept this article for publication at all.

\section{References}

1. Ministry of Health, Labour and Welfare Database in Japan [Internet]. Available from: https://www.mhlw.go.jp/toukei/saikin/hw/life/ tdfk15/dl/tdfk15-05.pdf

2. Haga Y, Ikei S, Ogawa M. Estimation of physiologic ability and surgical stress (E-PASS) as a new prediction scoring system for postoperative morbidity and mortality following elective gastrointestinal surgery. Surg Today. 1999 Mar; 29(3): 219-25.

3. Kubo H, Murayama Y, Arita T, et al. The prognostic value of preoperative neutrophil-to-lymphocyte ratio in colorectal cancer. World J Surg. 2016 Nov; 40(11): 2796-802.

4. Pozza A, Pauletti B, Scarpa M, et al. Prognostic role of neutrophil-to-lymphocyte ratio and platelet-to-lymphocyte ratio in patients with midgut neuroendocrine tumors undergoing resective surgery. Int J Colorectal Dis. 2019 Nov; 34(11): 1849-56.

5. Onodera T, Goseki N, Kosaki G. Prognostic nutritional index in gastrointestinal surgery of malnourished cancer patients. Nihon Geka Gakkai Zasshi. 1984 Sep; 85(9): 1001-5.

6. Jiang AG, Chen HL, Lu HY. The relationship between Glasgow prognostic score and serum tumor markers in patients with ad- vanced non-small cell lung cancer. BMC Cancer. 2015 Dec; 15: 386.

7. Miki C, Mohri Y, Toiyama Y, et al. Glasgow prognostic score as a predictive factor differentiating surgical site infection and remote infection following colorectal cancer surgery? Br J Cancer. 2009 Nov; 101(9): 1648-9; author reply 1650.

8. Pedrazzani C, Cerullo G, De Marco G, et al. Impact of age-related comorbidity on results of colorectal cancer surgery. World J Gastroenterol. 2009 Dec; 15(45): 5706.

9. Piso P, Bektas H, Werner U, et al. Comparison between treatment results for gastric cancer in younger and elderly patients. Zentralbl Chir. 2002 Apr; 127(4): 270-4.

10. Koppert LB, Lemmens VE, Coebergh JW, et al. Impact of age and co-morbidity on surgical resection rate and survival in patients with oesophageal and gastric cancer. Br J Surg. 2012 Dec; 99(12): 1693-700.

11. Basdanis G, Papadopoulos VN, Michalopoulos A, et al. Colorectal cancer in patients over 70 years of age: determinants of outcome. Tech Coloproctol. 2004 Nov; 8(1): s112-5.

12. Nitsche U, Spath $C$, Muller TC, et al. Colorectal cancer surgery remains effective with rising patient age. Int $\mathrm{J}$ Colorectal Dis. 2014 Aug; 29(8): 971-9.

13. Khan MR, Bari H, Zafar SN, et al. Impact of age on outcome after colorectal cancer surgery in the elderly-a developing country perspective. BMC Surg. 2011 Dec; 11(1): 17.

14. Gurlich R, Maruna $P$, Kalvach Z, et al. Colon resection in elderly patients: comparison of data of a single surgical department with collective data from the Czech Republic. Arch Gerontol Geriatr. 2005 Sep; 41(2): 183-90.

15. Bader TF. Colorectal cancer in patients older than 75 years of age. Dis Colon Rectum. 1986 Nov; 29(11): 728-32.

16. Banysch M, Akkaya T, Gurenko P, et al. Surgery for colorectal cancer in elderly patients: is there such a thing as being too old? G Chir. 2018 Nov; 39(6): 355-62.

17. Li JB, Luo S, Wong MCS, et al. Longitudinal associations between BMI change and the risks of colorectal cancer incidence, cancer-relate and all-cause mortality among 81,388 older adults : BMI change and the risks of colorectal cancer incidence and mortality. BMC Cancer. 2019 Dec; 19(1): 1082.

18. Bailon-Cuadrado M, Perez-Saborido B, Sanchez-Gonzalez J, et al. Prognostic nutritional index predicts morbidity after curative surgery for colorectal cancer. Cir Esp. 2019 Feb; 97(2): 71-80.

19. Sasaki M, Miyoshi N, Fujino S, et al. Development of novel prognostic prediction models including the prognostic nutritional index for patients with colorectal cancer after curative resection. J Anus Rectum Colon. 2019 Jul; 3(3): 106-15.

20. Ariake $\mathrm{K}$, Ueno $\mathrm{T}$, Takahashi $\mathrm{M}$, et al. E-PASS comprehensive risk score is a good predictor of postsurgical mortality from comorbid disease in elderly gastric cancer patients. J Surg Oncol. 2014 May; 109(6): 586-92.

21. Tominaga T, Takeshita H, Takagi K, et al. E-PASS score as a useful predictor of postoperative complications and mortality after colorectal surgery in elderly patients. Int J Colorectal Dis. 2016 Feb; 31(2): 217-25.

22. Yamamoto M, Saito H, Uejima C, et al. Estimation of physiological ability and surgical stress score is a useful prognostic indicator for elderly patients with colorectal cancer. Dig Surg. 2020; 37(2): 145-53.

23. Tomimaru Y, Ide Y, Murata K. Outcome of laparoscopic surgery 
for colon cancer in elderly patients. Asian J Endosc Surg. 2011 Feb; 4(1): 1-6.

24. Dekker JW, van den Broek CB, Bastiaannet E, et al. Importance of the first postoperative year in the prognosis of elderly colorectal cancer patients. Ann Surg Oncol. 2011 Jun; 18(6): 1533-9.
Journal of the Anus, Rectum and Colon is an Open Access journal distributed under the Creative Commons Attribution-NonCommercial-NoDerivatives 4.0 International License. To view the details of this license, please visit (https://creativ ecommons.org/licenses/by-nc-nd/4.0/). 